\title{
Li-Fi Technology: Increasing the Range of Li-Fi by Using Mirror
}

\author{
S. M. Tanvir Abid \\ Dept. of Computer Science, American International University-Bangladesh \\ Dhaka-1229, Bangladesh \\ E-mail: tanvirabid.aiub@gmail.com
}

\author{
Shiam Khabir, Md. Abir Hasan, Abhishek Saha, Md Masuduzzaman \\ Dept. of Computer Science, American International University-Bangladesh \\ Dhaka-1229, Bangladesh \\ E-mail: \{shiamkhabir, abirhasan.aiub, habhishek100,masuduzzaman.prince\}@ gmail.com
}

Received: 11 August 2018; Accepted: 22 September 2018; Published: 08 January 2019

\begin{abstract}
Data transmission is one of the most important term used in our day to day activities in the fast-growing world. Li-Fi opens a new era to that. Li-Fi is known as Light Fidelity. Simply it transmits data by visible light. According to recent research $\mathrm{Li}-\mathrm{Fi}$ has a range of approximately 10 meters. Also, it cannot pass through wall or any solid object. So, this research focuses mainly to increase the 10-meter range. For a regular sized room this range is enough. But if anyone wants to provide data inside a big hall or in any large room, it is not possible by only this range. He must provide more LED that is connected to the $\mathrm{Li}-\mathrm{Fi}$ router. This research focuses to optimize the number of LED and to minimize the cost. To increase the range of LED two methods are proposed here. One of the method deals with the positioning of the LEDs and another method is to use concave mirror. Smarter way of positioning of light gives better coverage of light that increases the range of LED. As concave mirror gives real and increased mirror for a particular positioning of an object, which is proved both theoretically and mathematically.
\end{abstract}

Index Terms-Light Emitting Diode (LED), Light Fidelity (Li-Fi), Mirror, Lenses, Range.

\section{INTRODUCTION}

In the era of data communication world, Li-Fi opens a new way of wireless communication. This technology uses LED lights to transmit data wirelessly. The existing wireless networks that connect us to the internet are very slow in various situations such as when multiple devices are connected. Also, when the number of devices is increased which access the internet, availability of fixed bandwidth makes it more difficult to use high data transfer rates and to be connected with a secure network. For data transfer Radio waves are just a small part of the electromagnetic spectrum available. On the other hand, Li-Fi has got a much larger spectrum for transmitting data compared to conventional methods of wireless communications which is depended on radio waves. The uncomplicated philosophy behind Li-Fi technology is that the data can be transmitted through LED light by varying light intensities which is faster than the human eyes can observe. This technology uses the Visible Spectrum which is a part of the electromagnetic spectrum that is still not significantly utilized, instead of Gigahertz radio waves for data transmission.

The idea of $\mathrm{Li}-\mathrm{Fi}$ was presented for the first time by a German physicist Harald Hass in the TED Global talk on 'Visible Light Communication (VLC)' in July 2011, by stating it as "data through illumination". Harald Hass used a table lamp made for LED bulb to transmit a video of a blooming flower which was then projected onto a screen. In simple terms, Li-Fi is thought of a light-based $\mathrm{Wi}-\mathrm{Fi}$ as an alternative of radio waves, it uses light to transmit data. Instead of $\mathrm{Wi}-\mathrm{Fi}$ modems, $\mathrm{Li}-\mathrm{Fi}$ uses transceivers fitted with LED lamps that could light a room as well as transmit and receive data and huge amount of information. Li-Fi can play a major role in relieving the heavy loads by adding new and unutilized bandwidth of visible light, to the currently available radio waves for data transfer that the current wireless system is facing. Therefore, it may compromise additional frequency band of the order of $410 \mathrm{THz}$ associated to that available in Radio Frequency communication which is about $300 \mathrm{GHz}$. Also, as the $\mathrm{Li}-\mathrm{Fi}$ uses the visible spectrum, it will help alleviate concerns that the electromagnetic waves coming with Wi-Fi could adversely affect our health. By Communication through visible light, Li-Fi technology has the possibility to change how we access the Internet, stream videos, receive emails and much more [1,2]. Security would not be an issue as data can't be accessed in the absence of light but the range of Li-Fi may be an issue.

Paper is organized as follows: section I introduces the LiFi technology and the limitation of its range. Section II gives the literature review as well as related works. Section III represents the methodology which is followed and the logics of the proposed solutions. In section IV 
mathematical calculations and analysis is given. Section $\mathrm{V}$ concludes the paper and discusses future works.

\section{RELATED WORKS}

Wi-Fi is very common medium of data transmission. It uses 2.4-5 GHz Radio Frequency (RF) to transmit data. Wi-Fi works when Internet or bandwidth is available. Without Internet or bandwidth Wi-Fi cannot transmit data. This is one of the main problem of Wi-Fi. Here speed limitations also major issue.

We tried to find out the solutions of this problem, to working on it we got modern technology which is Li-Fi. Li-Fi fixed the Wi-Fi problems and transmits data more accurately.

We studied different papers related Li-Fi Technology and got various problems and solutions. But we didn't get any solutions of Li-Fi range. We know, more range can cover large area of data transmission. So, we found that $\mathrm{Li}-\mathrm{Fi}$ range is one of the major issue while we tried to study on it and continue our research on Li-Fi range.

We proposed a new technique to increase $\mathrm{Li}-\mathrm{Fi}$ range. As there is no available research on this topic, we have faced many problems and we tried our best to solve those problems with suitable mathematical and geometrical solutions.

\section{A. Problems of Wireless Communication Systems}

Despite continuous improvements in wireless communication systems, (for example 4G, 5G, etc.) an impending crisis is predictable due to the lack of adequate Radio Frequency (RF) resources, this limitation in bandwidth can't support the progress in demand for high data rates and the large numbers of communication systems, within the bandwidths between $300 \mathrm{kHz}$ and 4 GHz. That's known as "Spectrum Crunch". [3]

Although, spectrum fulfillment shrinkages when high frequencies to transfer data is used, but this not a practice and proper solution, because this part of spectrum entails complex equipment and causes excessive cost systems. [4,5]

\section{B. So how does the problem can be solved}

Actually, there are various technologies that provide applicable and realistic solutions to this concern. One of them is the "Cognitive Radio". [6,7] It is a new category of wireless communication built with a transceiver architecture. It can perceptively detect which communication channels are in use and which are not, and immediately move into empty channels to use them to transmit data. Alternative solution is the transmission of data using visible light illumination. It uses very high frequency. This technology is known as Visible Light Communication (VLC). [6,7]

There are also many brilliant and efficient solutions, such as Light Fidelity (Li-Fi) technology, which is based on VLC.

\section{Visible Light Communication:}

Visible Light Communication is an optical communication technology that use visible light rays, these rays locate between [400-800] $\mathrm{THz}$, as visual carrier for data transmission by radiance. It uses fast pulsations of light. It cannot be perceived by the human eye. To transmit data. $[8,9,10]$ it includes any usage of the visible light portion of the electromagnetic variety to transmit information. The VLC standardization process is conducted within IEEE wireless personal area network working group (802.15) [11]. One of VLC ${ }^{c e}$ s features are providing wide bandwidth as illustrated in Figure 2. We can obviously see that usage the optical portion of spectrum guarantees about 10,000 times greater bandwidth compares to the usage of the RF frequencies. [12]

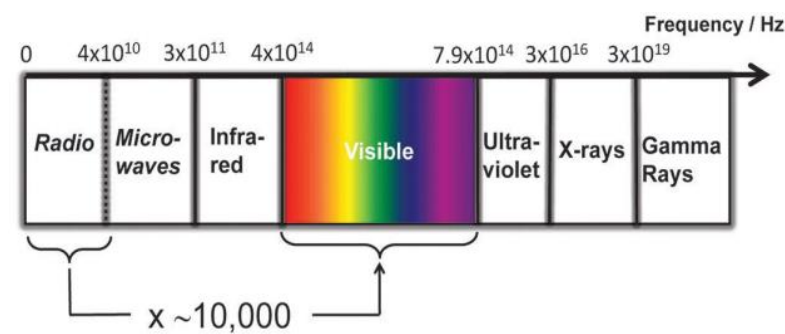

Fig.1. Location of visible light and RF frequencies at electromagnetic spectrum

\section{Li-Fi Technology : Challenges}

$\mathrm{Li}-\mathrm{Fi}$ technology is facing some problems such as

$\checkmark \quad$ Li-Fi entails line of sight (LOS) and the receiving device can't be shift in indoors.

$\checkmark$ A key challenge is how the receiving device Li-Fi Technology will transmit the data back to transmitter.

$\checkmark$ Another significant issue is intrusion from external light sources like normal bulbs, sun light; dense materials in the path of transmission will cause disruption in the communication.

$\checkmark$ Another disadvantage is Li-Fi doesn't work in the dark. As light can't pass through objects, so if the receiver is inadvertently blocked in any way, then the signal will instantly cut out. And the signal might be easily blocked by someone simply walking in front of the LED source. $[13,14]$

\section{What we want to do}

We mainly focused on increasing the range of LED. For increasing the LED, we able to decreases cost and better performance of Li-Fi system. For which we can use less amount of LED for certain area from previous architecture.

\section{PROPOSED METHOD}

This research seeks to improve the range of existing Li-Fi technology. So, the research topic refers to applied research or action research category. To improve the range, the proposal is to use concave mirror to develop the visible light range by increasing the distance and the 
angle. That indicates our background knowledge should be electromagnetic waves, light properties, mirrors and lens. There are no existing techniques of increasing Li-Fi range. We will propose a new technique. For implanting the system, we propose to use concave mirror which is able to reduce the number of LEDs as well as increase their range efficiently.

From various resources done in literature review chapter it was mentioned that if the intensity of LEDs is increased, it increases the range as well. So, we focused on intensity and found some ways as -

- $\quad$ Supplying more current to the LEDs

- Supplying more voltage to the LEDs

But, both these methods decrease the LED life.

Other ways to increase the intensity are

- Using lens

- Using mirrors

We choose mirror over lens because of its availability and less cost.

A. Ideas of using mirror in various way:

As mirrors reflects light comes from an object, we may use the mirror by setting them in an efficient angle, so that the mirrors can reflect the light which came from LED and that can be received by another mirror and so on.

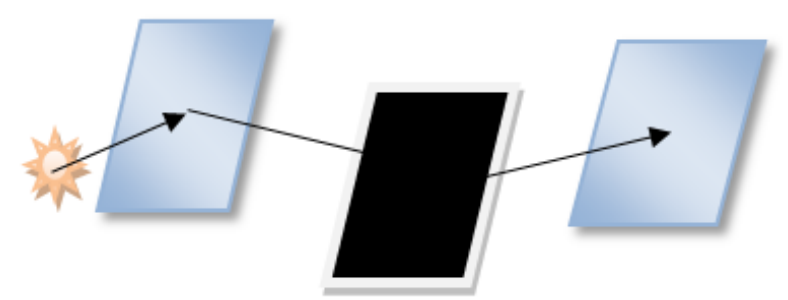

Fig.2. Reflection of light- mirror to mirror

But there are some problems in this method as finding the right angle and it is complicated to calculate intensity of light increased in the area.

$>$ Another way is to use the spherical mirrors. which are

- Convex mirror.

- Concave mirror

Convex mirror

Convex mirror never gives any real image

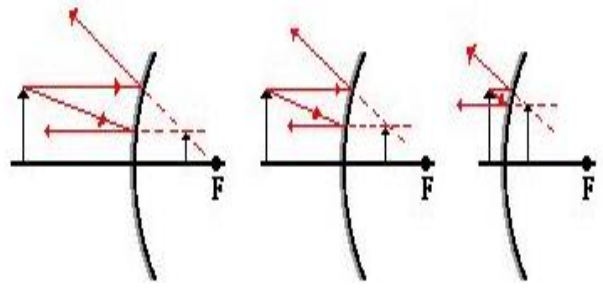

Fig.3. Image of Convex mirror is

The diagrams above show that in each case, the image

- located behind the convex mirror

- a virtual image

○ an upright image

$\circ$ reduced in size

\section{Concave Mirror}

Concave mirror gives real and extended image when object is located between center of curvature and main focus

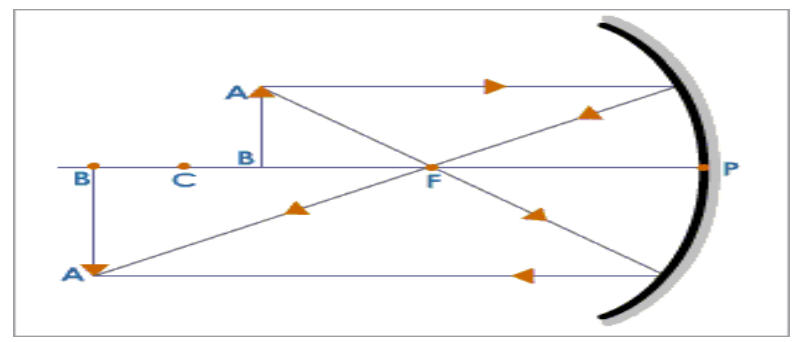

Fig.4. Image of Concave mirror is

The diagrams above show that in each case, the image

- Located front the concave mirror.

- A real image.

- A downright image.

- Increased in size.

Electromagnetic wave: Light

The intensity of light is given by pointing vector. It defines the power output per unit area $\left(\mathrm{w} / \mathrm{m}^{2}\right)$ of the electromagnetic wave. Simply it is the changing rate of energy per unit area of any electromagnetic wave.

Luminous Flux and Luminous intensity:

Luminous flux is the measure of perceived power of visible light. Unit is lumens (lm). Simply it is the quantity that tells us how much power a certain light wave carries.

$6831 \mathrm{~m}=1 \mathrm{watt}$ of power carried by light of wave length $555 \mathrm{~nm}$. 
Luminous intensity defined as the luminous flux per solid angle. Luminous intensity expresses the directionality of the energy emitted by the light.

Solid angle: This is defined as two-dimensional angle define using units knows as steadies.

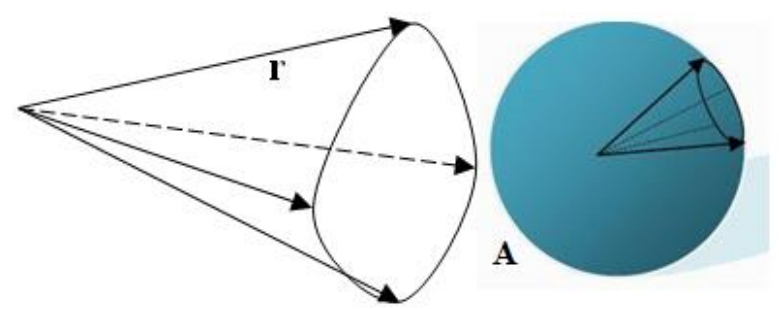

Fig.5. Solid angle and solid angle in a sphere

$$
\text { Solid angle }=\frac{\text { Area }}{r^{2}}
$$

Where $\mathbf{A}$ is the surface area on a sphere with radios $\mathbf{r}$ Luminous intensity can be defined as luminous flux/solid angle.

\section{Light Projection:}

It is well known that, visible light projects as a cone. And the more is the distance is less the intensity. Now, considering a light source without a mirror behind. The source. So simply we can consider it as a cone. With the use of a mirror we may able to expand the angle.

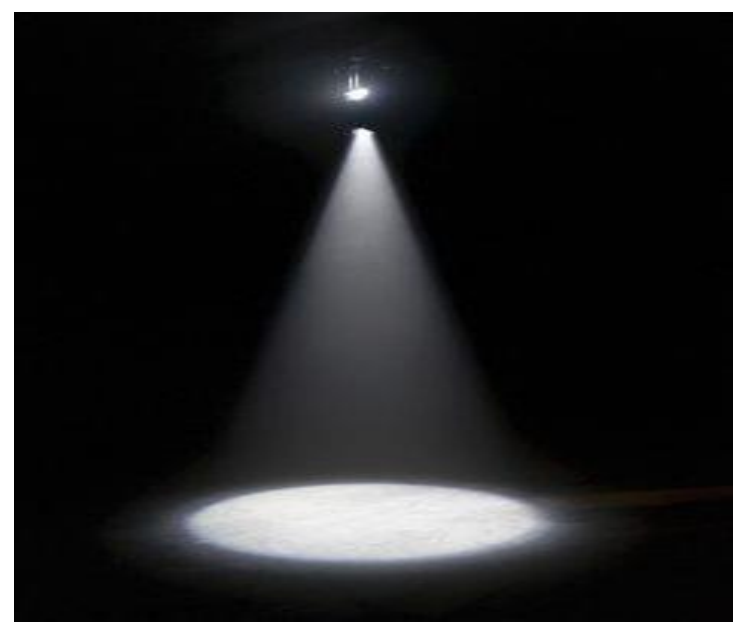

Fig.6. Light Projection

Now there are 2 things to be concerned,

- What happened if the angle is expended

- How the angle can be expended

As we know from the definition of solid angle,

$$
\text { Angle }=\frac{\text { Area }}{r^{2}}
$$

So, from equation we can say area increases when the angle increases. That means if angle can be expanded, then it is possible to cover more area.

To expand the angle, we propose to use concave mirror. As mentioned before when the object is situated in between center of curvature and main focus, it gives inverted but a real image which is increased in size. So, if we place the LED in between center of curvature and main focus of the mirror, it will give an expanded real image of the LED. As a bigger LED has more intensity as well as the angle of its light projection is bigger, this automatically increases the range of normal LED.

\section{B. Increasing Range by Changing Position of LED:}

According to the usual setup of household lights Li-Fi might be set in the ceiling connected to the $\mathrm{Li}-\mathrm{Fi}$ router. Concept of light projection tells that it will make cone shape from the ceiling.

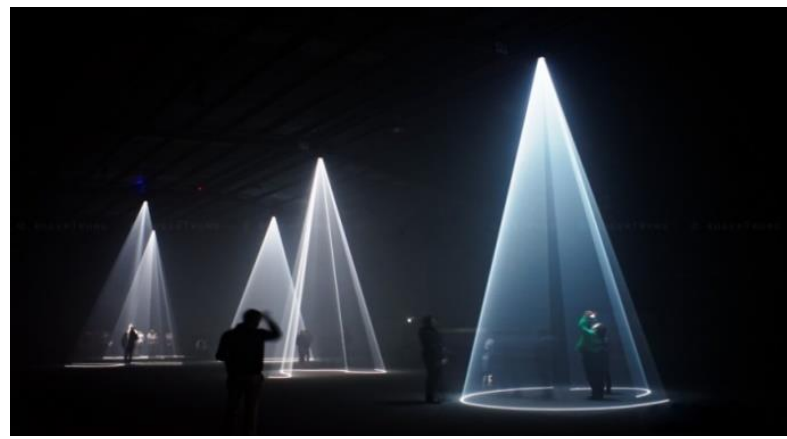

Fig.7. Light projection as a cone from ceiling

We propose another way to set the light connected with the router. The lights must be set on the vertical wall of a regular rectangular room so that less LED can cover the whole room by projecting same amount of light.

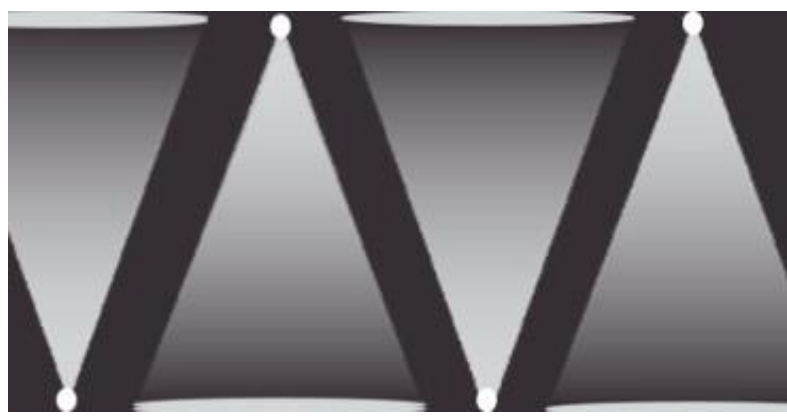

Fig.8. Number of LED can be reduced by positioning them in optimistic way- A view from the ceiling

C. How method of expanding angle can be a better solution:

As we saw if LEDs are set in the optimistic way, number of LED is decreased. Now according to the properties of concave mirror we get a real increased image as well as greater angle than the real LED, so using concave mirror we will get greater angle. As mentioned before

$$
\text { Area }=\text { angle } \times r^{2}
$$


That means greater the angle is, greater the area will be.

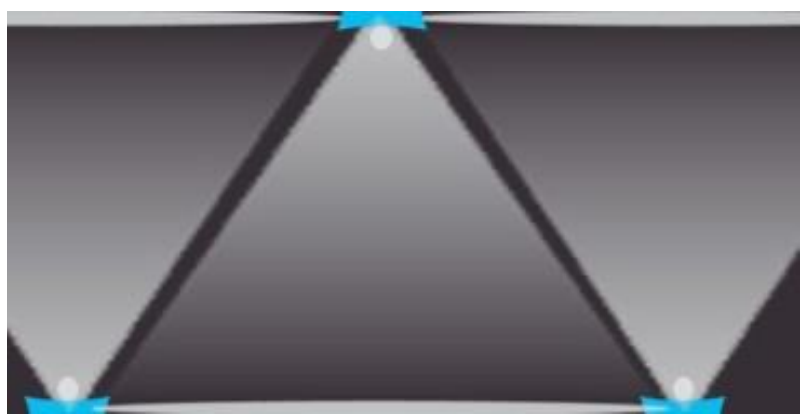

Fig.9. Angle and area is increased using concave mirror-A view from the ceiling

So, expanding the angle and area it reduces the number of LED again. Now the same area can be covered by using lesser number of LEDs than before.

Though according to the theory, light projects as shown in the figures, but in real life the darker areas beside the cone remains no longer dark because of reflection from surroundings.

\section{CAlculation Results and ANAlysis}

\section{A. Concave mirror gives increased image located ahead of actual LED:} $10 \mathrm{~cm}$

Let us assume a concave mirror of focal length $\mathrm{f}=$

So that the distance of center of curvature

$$
\mathrm{C}=2 \mathrm{f}=2 \times 10=20 \mathrm{~cm}
$$

According to the properties of concave mirror, to get real and bigger image, LED has to be set in between the center of curvature and the main focus,

Let the distance of LED is placed $u=18 \mathrm{~cm}$. LED size $\mathrm{h}=9 \mathrm{~cm}$. So if the image size is $\mathrm{h}$ ' and the distance of image is $v$, then from magnification we know

$$
m=\frac{h^{\prime}}{h}=-\frac{v}{u}
$$

Now, to find the distance $\mathrm{v}$, we know

$$
\begin{aligned}
& \frac{1}{v}+\frac{1}{u}=\frac{1}{f} \\
& \text { or } \frac{1}{v}=\frac{1}{f}-\frac{1}{u} \\
& \text { or } \frac{1}{v}=\frac{1}{10}-\frac{1}{18} \\
& \text { or } \frac{1}{v}=0.0444 \\
& v=22.50
\end{aligned}
$$

So, the distance of image is $22.5 \mathrm{~cm}$ which is $4.5 \mathrm{~cm}$ ahead of the LED.

Now, placing the value of $v, u$ and $h$ in (1) we get,

$$
\begin{aligned}
& \frac{h^{\prime}}{h}=-\frac{v}{u} \\
& \Rightarrow h^{\prime}=-\frac{v h}{u} \\
& \Rightarrow h^{\prime}=-\frac{22.50 \times 9}{18} \\
& \Rightarrow h^{\prime}=-11.30
\end{aligned}
$$

Here the - (minus) sign represents that the image in inverted.

So, the Image height is $11.30 \mathrm{~cm}$ which is $2.30 \mathrm{~cm}$ bigger than the real LED.

As we know a bigger LED can project light with a greater angle than the smaller one. Also, a bigger LED has more intensity than a smaller one. So, the increased image of LED will give more angle and intensity.

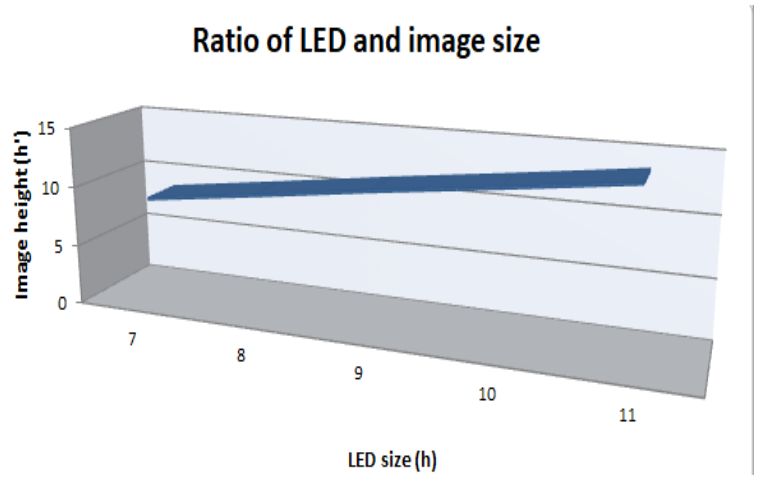

Fig.10. Ratio of LED and Image size

From the graph we can see the height of the image remains larger than the actual LED size.

Also, here the increased image is built $3.5 \mathrm{~cm}$ ahead of the real LED, so it also increased the distance covered by the LDE. Simply it increases the range.

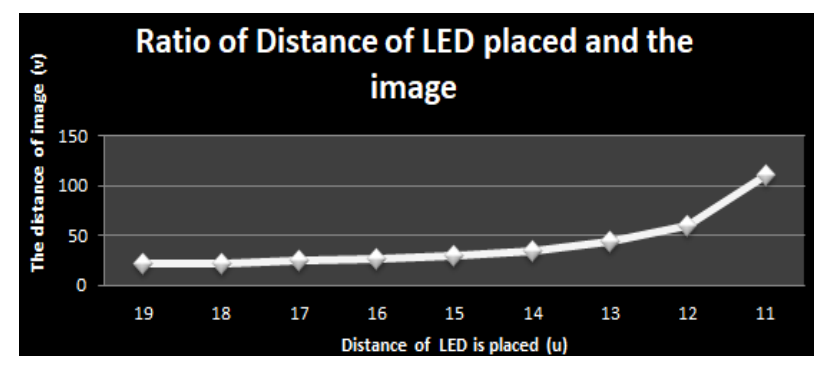

Fig.11. Ratio of distance of LED placed and the image

From the graph we can see the concave mirror always gives greater distance than the place where LED is placed.

B. How greater angle can cover greater area:

As it is known from the characteristics of light and light projection, it is a cone shape inside a sphere of radius $\mathrm{R}$ which is also the side of the cone.

If the height of the cone is $\mathbf{h}$ and the radius of the base 
is $\mathbf{r}$, then the volume of cone

$$
V=\frac{\pi r^{2} h}{3}
$$

Base area of a cone is a $2 \mathrm{D}$ circle of radius $r$. If the angle increases, so does the radius of the base.

Let's assume 2 cones with the same height but different angle, that means different base radius.
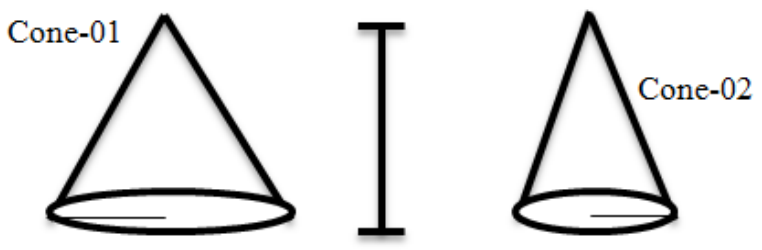

Fig.12. Two cones with different angle

For cone 01 , let's assume,

Height $\mathrm{h}=14 \mathrm{~cm}$

Base radius $\mathrm{r}=5 \mathrm{~cm}$

$A=\pi r^{2}$

$\Rightarrow A=3.1416 \times 5^{2}$

$\Rightarrow A=78.84 \mathrm{~cm}^{2}$

And volume $V=\frac{\pi r^{2} h}{3}$

$V=\frac{3.1416 \times 5^{2} \times 14}{3}$

$\Rightarrow V=366.52 \mathrm{~cm}^{3}$

Again, for cone 02 , as the height remains the same, so

$$
\text { Height } \mathrm{h}=14 \mathrm{~cm}
$$

Base radius $r=2.5 \mathrm{~cm}$

$$
\begin{gathered}
\text { So, area } A=\pi r^{2} \\
\Rightarrow A=3.1416 \times 2.5^{2} \\
\Rightarrow A=19.635 \mathrm{~cm}^{2} \\
\text { And volume } V=\frac{\pi r^{2} h}{3} \\
V=\frac{3.1416 \times 2.5^{2} \times 14}{3} \\
\Rightarrow V=91.63 \mathrm{~cm}^{3}
\end{gathered}
$$

From this calculation we can see if the angle is increased, area as well as the volume both also increases. That means, if we use mirror to get greater angle then when the angle increases, it covers more area or in $3 \mathrm{D}$ it covers more space of a room. It automatically increases the range of $\mathrm{Li}-\mathrm{Fi}$.

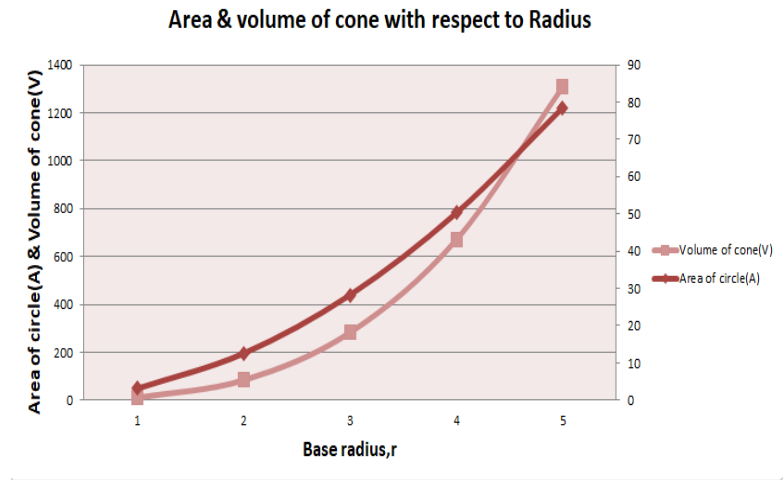

Fig.13. Area and volume of cone with respect to surface radius

From the graph above we can see area and volume increases when radius so that the angle increases. So, it is clear if we increase angle, it can cover more area.

\section{FUTURE WORK \& CONCLUSION}

In present Li-Fi technology uses light that cannot go outside of any room or cannot pass any solid object that stops light. In future research this limitation can be considered, and a solution can be found regarding this. Also, our proposal of increasing the range of light can be proved by implementing in real life. Also, it can be tested that if our inverted image of LED gives any distorted data or not. Combination of lens and mirror might make the intensity more than only use of mirrors.

As the range of Li-Fi is limited now days and soon it will be the broadly used technology, it is really important to increase the range of $\mathrm{Li}-\mathrm{Fi}$. As we proposed to use a concave mirror and the placing position of LED it will increase the range of each LED individually. Smarter way of positioning of light gives better coverage of light that increases the range of LED. Concave mirror gives larger image with greater angle which increases area that is covered by the light and the image created ahead of the LED which also increases the horizontal range of the light. Though it may not possible now days to increase the range from one room to another, but the method will help us to cover an enormous sized room.

\section{REFERENCES}

[1] Shubham Panjwani, Abhinav, Aishwarya Varshney, "LiFi:Internet Through Light," International Journal of Computer \& Mathematical Sciences, Volume 6, Issue 3,March 2017

[2] Study Paper on LiFi (Light Fidelity) and its Applications[online] http://tec.gov.in/pdf/Studypaper/lifi\%20study\%20paper\% 20-\%20approved.pdf (2013)

[3] Enhanced data transmission protocol for visible light communications.[Online] http://www.researchinnovation.ed.ac.uk/Opportunities/enhanced-datatransmission-for-Li-Fi-communications.aspx $(2015$, jan) 
[4] Dimitrov, S. and Haas, H. "Principles of LED Light Communications Towards Networked Li-Fi," Cambridge, United Kingdom: Cambridge University Press, 2015

[5] TED[Online]. http://www.ted.com/talks/harald_haas_wireless_data_fro m_every_light_bulb (2011,Jul)

[6] H. Anandakumar \& K. Umamaheswari, "An Efficient Optimized Handover in Cognitive Radio Networks using Cooperative Spectrum Sensing," Intelligent Automation \& Soft Computing, 2017

[7] Techtarget search networking. [Online] http://searchnetworking.techtarget.com/definition/cognitiv e-radio (2010)

[8] Prerna Chauhan, Ritika Tripathi Jyoti Rani, "Li-Fi (Light Fidelity)-The future technology In Wireless communication," International Journal of Applied Engineering Research, Nov 2012.

[9] Anurag Sarkar and Prof. Shalabh Agarwal, "Li-Fi technology: Data transmission through visible light," Advance Research in Computer Science and Management Studies. June 2015.

[10] M. Mutthamma, "A survey on Transmission of data through illumination - Li-Fi," International Journal of Research in Computer and Communication Technology, Vol 2, Issue12,December- 2013.

[11] Wikipedia.[Online]. http://en.wikipedia.org/wiki/Visible_light_communication (2015)

[12] Kajal, Ashish Saini Kanchan Gupta, "Li-Fi- Light Fidelity Technology- A Review," International Journal of Emerging Research in Management \&Technology, vol. 3, no. 10, Oct 2014.

[13] Raunak, Akshay Sanganal, Rahul R. Sharma, "Li-Fi Technology Transmission of data through light," Int.J.Computer Technology \& Applications, Navi Mumbai, India, 2014.

[14] Prof. (Dr.) Y.P.Singh, "A Comparative and Critical technical Study of the Li-Fi - (A Future Communication) $\mathrm{V} / \mathrm{S}$ Wi-Fi," International Journal of IT, Engineering and Applied Sciences Research (IJIEASR), vol. 2, April 2013.

\section{Authors' Profiles}

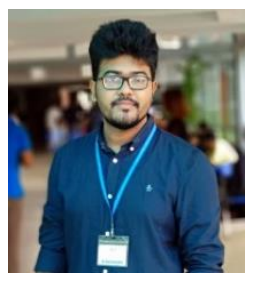

S. M. Tanvir Abid is a citizen of Bangladesh and has received his bachelor's degree in Computer science \& Engineering (CSE) in 2018 from American International UniversityBangladesh (AIUB). He has completed his Internship as Teaching Assistant in American International UniversityBangladesh (AIUB). Also he is working as graphics design course instructor in an institute named ICT Career. Currently he is applying to different European universities to complete his master's degree. His research interest includes wireless sensor network, artificial intelligence, network security, virtual reality and cloud computing. He is also involved in scouting and some different voluntary works in his country.

LinkedIn Profile: https://www.linkedin.com/in/its-tanvir-abid/

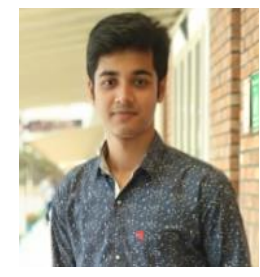

Shiam Khabir who is completed his B.Sc. degree in Computer Science \& Engineering (CSE) in 2018 from American International UniversityBangladesh (AIUB). Now he is applying different foreign university to complete his masters. At the same time, he tries for a Govt. IT Job as well. His research interest includes wireless sensor network, virtual reality and network security.

LinkedIn Profile: https://www.linkedin.com/in/shiam-khabir9b381ab7/

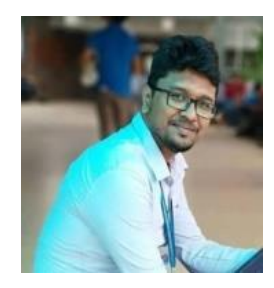

Md. Abir Hasan is a citizen of Bangladesh and Completed his B.Sc. degree in Computer Science \& Engineering (CSE) in 2018 from American International UniversityBangladesh (AIUB). He has completed his Internship at Guardian IT Ltd (an IT company of Bangladesh). Now he is applying different foreign and local university to do his postgraduation. At the same time, he is working as an IT Project Manager at IT Grow Division Ltd (a software company of Bangladesh). His areas of interest include Computer networks and architecture, Information and Network Security, Wireless Communications and Security, Cloud Computing, data management, algorithms and artificial intelligence. He is also involved in some different voluntary work in his country.

LinkedIn Profile: https://www.linkedin.com/in/itsabirhasan/

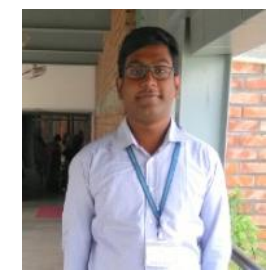

Abhishek Saha is a Bangladeshi and $\mathrm{He}$ Completed his B.Sc. degree in Computer Science \& Engineering (CSE) in 2018 from American International UniversityBangladesh (AIUB). He has completed his Internship from AIUB as a Teacher Assistant. He Has a good hands-on experience in Oracle and SQL Server. Now he is applying for Germany to do his post-graduation. Currently, $\mathrm{He}$ is working also as a software engineer in a IT Company. He like to work in Computer networks related problem, Information and Network Security, Cloud Computing, database management, algorithms and artificial intelligence. He is also connected with voluntary work in his country.

LinkedIn Profile: https://www.linkedin.com/in/abhishek-saha4b16b198/

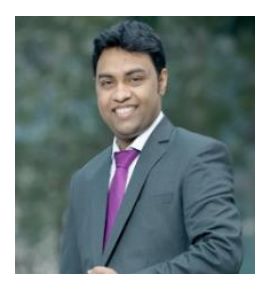

Md. Masuduzzaman is a citizen of Bangladesh and currently working as a lecturer in the dept. of Computer Science at American International UniversityBangladesh (AIUB). He has received his Masters degree specialized in Computer Networks and Architecture in 2015 and received his Bachelor degree in Computer Science and Engineering in 2013 from American International University-Bangladesh (AIUB). His areas of interest includes Computer netwoks and architecture, Information and Network 
Security,Cloud Computing and Internet of Things (IoT). He is also involved in some different sports sector and voluntary work in his country.

LinkedIn Profile: https://www.linkedin.com/in/masud-prince/

How to cite this paper: S. M. Tanvir Abid, Shiam Khabir, Abir Hasan, Abhishek Saha, Masuduzzaman, "Li-Fi Technology: Increasing the Range of Li-Fi by Using Mirror", International Journal of Information Technology and Computer Science(IJITCS), Vol.11, No.1, pp.50-57, 2019. DOI: 10.5815/ijitcs.2019.01.06 